Notfall Rettungsmed 2021 $24: 826-830$ https://doi.org/10.1007/s10049-021-00920-x Angenommen: 18. Juni 2021

Online publiziert: 13. Juli 2021

๑) Springer Medizin Verlag GmbH, ein Teil von Springer Nature 2021

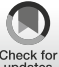

\section{Qualitätskriterien und strukturelle Voraussetzungen für Cardiac Arrest Zentren - Update 2021}

\author{
Deutscher Rat für Wiederbelebung/German Resuscitation \\ Council (GRC)
}

\title{
Zusammenfassung
}

Es handelt sich hierbei um ein Update der im Frühjahr 2017 erstmals beschriebenen Qualitätskriterien und strukturellen Voraussetzungen für Cardiac Arrest Zentren auf Basis der ersten Zertifizierungen und Erfahrungen. Die Kriterien wurden angepasst und konkretisiert und zum Teil zur Umsetzbarkeit im klinischen Alltag neu definiert.

Schlüsselwörter

Postreanimationsbehandlung · Cardiac Arrest Zentrum - Rettungskette - OHCA - außerklinischer Herz-Kreislaufstillstand · Lebensrettende Systeme

\section{Einleitung}

Im Frühjahr 2017 wurde durch ein multiprofessionelles Expertengremium im Auftrag des Deutschen Rates für Wiederbelebung/German Resuscitation Council (GRC) ein Konsensuspapier erstellt, in dem erstmals Qualitätskriterien und strukturelle Voraussetzungen für Cardiac Arrest Zentren beschrieben wurden [1-4]. Auf Basis dieser Kriterien wurden seit Ende 2018 bis Sommer 2019 erste Audits an 8 ausgewählten Pilotkliniken vorgenommen, und zwischenzeitlich konnten bis Ende des Jahres 2020 insgesamt 57 Kliniken erfolgreich als Cardiac Arrest Zentren zertifiziert werden [5].
Die Zertifizierungen (• Abb. 1) werden durch eine Zertifizierungsgesellschaft unter Aufsicht eines Kuratoriums durchgeführt. Das Kuratorium besteht aus Mitgliedern des GRC und der Deutschen Gesellschaft für Kardiologie - Herz- und Kreislaufforschung e. V. (DGK).

Auf Basis der ersten Erfahrungen wurden im Verlauf die Kriterien angepasst und konkretisiert und zum Teil zur Umsetzbarkeit im klinischen Alltag neu definiert.

Wesentliche Neuerungen im Kriterienkatalog betreffen die Forderung nach $\mathrm{Er}$ stellung einer Geschäftsordnung für Cardiac Arrest Zentren, die Rolle des Cardiac Arrest Receiving Teams im Cardiac Arrest Zentrum, die Definition der geforderten Kooperation mit der Neurologie und die 
Schulung der Mitarbeiter in zertifizierten Kursen sowie die Umsetzung der Weiterbildungskonzepte.

In der vorliegenden überarbeiteten Version finden diese Punkte Berücksichtigung.

Selbstverständlich sollen in Pandemiezeiten (z.B. COVID-19) die lokalen gültigen Regularien und ggf. entsprechend adaptierte Vorgaben berücksichtigt werden.

Es handelt sich um ein lernendes System, die Kriterien werden auch künftig weiter überarbeitet. Das Kuratorium ist an Feedback und Verbesserungsvorschlägen interessiert.

Aus Gründen der besseren Lesbarkeit wird auf die gleichzeitige Verwendung der Sprachformen männlich, weiblich und divers $(\mathrm{m} / \mathrm{w} / \mathrm{d})$ verzichtet. Sämtliche Personenbezeichnungen gelten gleichermaßen für alle Geschlechter.

\section{Ursachen eines prähospitalen Kreislaufstillstands}

Die Ursachen eines prähospitalen Kreislaufstillstands sind vielfältig, und es sollte zwischen kardialen und nichtkardialen Ursachen unterschieden werden. Neben kardialen Ursachen, wie dem akuten Myokardinfarkt oder einer primären Herzrhythmusstörung, sind Lungenembolien, Intoxikationen, zerebrale oder metabolische Ereignisse, Traumata, schwere Schockzustände oder eine primäre Hypoxie als Ursache für einen Kreislaufstillstand aufzuführen.

\section{Intrahospitale Weiterversorgung in spezialisierten Zentren [1]}

Die Prognose reanimierter Patienten ist - neben der therapiefreien Zeit - im Wesentlichen davon abhängig, wie schnell und mit welcher Qualität die zur Reanimation führende Ursache behandelt wird. So ist bei reanimierten Patienten mit akutem ST-Hebungs-Infarkt innerhalb von maximal 90 bis 120 min ab Erstkontakt mit dem Rettungsdienst die Rekanalisation des verschlossenen Kranzgefäßes empfohlen [6, 7].

Bei reanimierten Patienten handelt es sich nicht selten um klinisch ausgesprochen instabile Patienten, die in der frühen Phase Komplikationen (z. B. kardiogener Schock, sepsisähnliche Zustandsbil- der, Nierenversagen, Beatmungsprobleme etc.) erleiden und bei denen im weiteren Verlauf Entscheidungen mit hoher fachspezifischer Kompetenz (z. B. Herzinsuffizienztherapie, Defibrillatorimplantation) getroffen und umgesetzt werden müssen. Auch muss sich schon früh ein langwieriger Rehabilitationsprozess mit aufwendigen physio-, aber auch psychotherapeutischen Maßnahmen anschließen.

Die Betreuung dieses Krankheitsbildes setzt daher eine extrem hohe spezifische Qualifikation der einzelnen beteiligten Fachdisziplinen voraus, die nicht nur für sich genommen eine große Erfahrung im Umgang mit reanimierten Patienten haben müssen (Versorgung hoher Fallzahlen), sondern sich auch durch eine enge interdisziplinäre Kooperation auszeichnen. Reanimierte Patienten werden dort am besten versorgt, wo viele dieser Patienten behandelt werden.

Daher empfehlen die internationalen Leitlinien die Behandlung reanimierter Patienten in sogenannten Reanimationsbzw. Cardiac Arrest Zentren, die sich strukturell, organisatorisch und logistisch auf die Versorgung speziell dieser Patienten ausgerichtet haben [8-10].

\section{GRC-Kriterien für Cardiac Arrest Zentren}

Eine standardisierte Postreanimationsbehandlung sollte in Cardiac Arrest Zentren überprüfbar garantiert sein. Nachfolgend werden Qualitätskriterien zur Krankenhausversorgung von Patienten nach außerklinischem Kreislaufstillstand aufgeführt. Sie basieren auf einem evidenzbasierten Expertenkonsens der interdisziplinären Arbeitsgruppe "Cardiac Arrest Zentren" des GRC und einer Überarbeitung des Kuratoriums [1].

Nach den Erfahrungen aus den ersten Audits bis Ende des Jahres 2020 wurden diese Kriterien jetzt überarbeitet und aktualisiert.

\section{Allgemeine Voraussetzungen}

1. Erstellung einer Geschäftsordnung mit Definition der Zusammensetzung und der Leitung des Cardiac Arrest Zentrums.
2. Vorhandensein folgender Fachdisziplinen mit konsekutivem Leistungsspektrum: interventionelle Kardiologie, Anästhesiologie, Neurologie, fachgebietsbezogene Intensiv- und Notfallmedizin, Unfallchirurgie (in den Zentren, in denen Traumapatienten aufgenommen werden; [1])

3. Minimal gefordert ist die Möglichkeit der Durchführung folgender Maßnahmen: Herzkatheterdiagnostik und $\mathrm{PCl}$, radiologische Bildgebung inklusive der Möglichkeit der Ganzkörper-CT, Temperaturmanagement, Echokardiographie inkl. transösophageale Echokardiographie, Notfalldialyse.

4. Standardisierte Behandlungspfade für reanimierte Patienten mit unterschiedlicher Ursache, wie sie z. B. für Patienten mit ST-Hebungs-Infarkt und/ oder Traumapatienten existieren [1]

5. Standardisierte Protokollierung der zeitlichen Abläufe [1]

Neben diesen allgemeinen Voraussetzungen ergeben sich folgende Anforderungen an die Struktur-, Prozess- und Ergebnisqualität:

\section{Strukturqualität}

1. 24/7-Verfügbarkeit einer geeigneten Übernahmeeinrichtung [1]

Als geeignete Notaufnahme/ Übernahmeeinrichtung können dienen: Schockraum, Reanimationsraum, Notaufnahmeraum einer Intensivstation und Herzkatheterlabor. Unabdingbar ist die vollständige Verfügbarkeit aller für die Intensivtherapie erforderlichen Ausstattungen und der entsprechenden personellen Ressourcen.

2. 24/7-Verfügbarkeit eines definierten Cardiac Arrest Receiving Teams Ziel ist es, Patienten nach erfolgreicher Reanimation bereits bei Übernahme in der Klinik unmittelbar standardisierten Abläufen mit zuvor festgelegten diagnostischen und therapeutischen Maßnahmen zuzuführen und dies durch ein erfahrenes interprofessionelles Übernahmeteam (Cardiac Arrest Receiving Team [CART]) mit entsprechender Personalstärke sicherzustellen. Aus Sicht 
des Kuratoriums muss das CART aus mindestens 2 Ärzten (darunter möglichst einem Facharzt) sowie aus 2 Pflegekräften aus den Bereichen Intensivmedizin/Notfallmedizin bestehen [11].

3. Herzkatheterlabor mit 24/7-PCIVerfügbarkeit [1]

Die Zentren müssen eine 24-hRufbereitschaft für die unmittelbare Durchführung einer Notfall-PCI (perkutane Koronarintervention) garantieren. Das Personal muss innerhalb von 30 min nach Alarmierung im Katheterlabor präsent sein. Es müssen Ablaufprotokolle entsprechend den Leitlinien für die STEMI-Behandlung [6] und die Non-STEMI-Behandlung [12] bestehen, und die zeitlichen Abläufe der Infarktbehandlung müssen überprüfbar dokumentiert werden. Die Möglichkeit der Direktübergabe reanimierter STEMI-Patienten durch den Rettungsdienst im Katheterlabor muss jederzeit akut gewährleistet sein [13].

Je Cardiac Arrest Zentrum sind für die Gewährleistung des 24/7-Bereitschaftsdiensts mindestens vier erfahrene Interventionskardiologen erforderlich. Die an dem Rufdienst teilnehmenden Interventionskardiologen sollten einen zertifizierten Advanced-Life-Support(ALS)-Kurs (oder einen vergleichbaren zertifizierten Kurs) absolviert haben, und mindestens eine Person aus dem Team des Herzkatheterlabors sollte an einem zertifizierten ALSoder Intermediate-Life-Support(ILS)Kurs (oder einem vergleichbaren zertifizierten Kurs) erfolgreich teilgenommen haben und muss für das kontinuierliche Weiterbildungskonzept des gesamten Teams des Katheterlabors verantwortlich sein (neben dem Leiter des CAC).

4. 24/7-Verfügbarkeit der Notfallsonographie [1]

Eine jederzeitige Möglichkeit der Notfallsonographie der Herz-ThoraxOrgane sowie des Abdomens, z. B. anhand der DEGUM-Empfehlungen, ist unabdingbar. Die Durchführung der Sonographie muss bei jedem Patienten nach prähospitalem Kreislauf- stillstand auch in der Rufbereitschaft gewährleistet sein.

5. 24/7-Verfügbarkeit von Notfallröntgen und Computertomographie (CT; [1])

6. 24/7-Verfügbarkeit eines Platzes auf einer Intensivstation inklusive der Ausstattung zum leitliniengerechten Temperaturmanagement [1] Pro Schicht sollte zumindest der diensthabende Arzt der Intensivstation einen zertifizierten ALS-Kurs (oder einen vergleichbaren zertifizierten Kurs) absolviert haben, und mindestens eine Person aus dem Assistenzteam sollte an einem zertifizierten ILS-Kurs (oder einem vergleichbaren zertifizierten Kurs) teilgenommen haben.

Das Personal muss bezüglich des Temperaturmanagements geschult sein.

7. 24/7-Verfügbarkeit eines fachneurologischen Dienstes

Dies gilt sowohl für das Vorhandensein einer neurologischen Fachabteilung (bzw. Belegabteilung) im Hause, wie auch für den Fall einer Kooperationsvereinbarung mit einer Fachabteilung für Neurologie an einer externen Institution. Hier sollen „rund um die Uhr" (24/7) folgende Kriterien erfüllt sein:

a. Ein direkter Telefonkontakt mit einem Facharzt für Neurologie muss innerhalb von 30 min garantiert möglich sein.

b. Die Möglichkeit einer teleradiologischen CT- und MRT-Befundung muss innerhalb von 30 min garantiert sein.

c. Die Möglichkeit einer Akutvisite am Bett durch einen Facharzt für Neurologie muss bei besonders komplexen Fällen innerhalb von 30 min garantiert sein.

8. Regelmäßige Qualitätszirkel zur Reanimationsversorgung [1] Der Nachweis eines regelmäßigen lokalen Qualitätszirkels zur Reanimationsversorgung ist erforderlich. Es sollen hier alle Komponenten und Disziplinen der präklinischen und klinischen Versorgungskette eingebunden sein. Der Zirkel muss systematisch und regelmäßig statt- finden (mind. 1x/Jahr) und neben einer Analyse der Prozess- und Ergebnisqualität auch Einzelfallanalysen beinhalten.

9. Schulung von Mitarbeitern in Cardiac Arrest Zentren in zertifizierten Kursen Im Positionspapier "Qualitätsindikatoren und strukturelle Voraussetzungen für Cardiac Arrest Zentren" des GRC wurde eine entsprechende Schulung in zertifizierten Kursen festgeschrieben (ALS/ACLS- und ILSKurse). Mit Beginn der Zertifizierung stellte sich die Frage, wie genau die beschriebenen Anforderungen in den Kliniken nachhaltig umgesetzt werden können.

Das Kuratorium für die Zertifizierung der Cardiac Arrest Zentren hat daher für die Erstauditierung festgelegt, dass der fehlende Nachweis zertifizierter Kurse zunächst kein Ausschlusskriterium für die Erteilung eines Zertifikats darstellt.

Für die praktische Umsetzung gilt die folgende Vorgehensweise:

Im Erstaudit erfolgt an die zu zertifizierende Klinik der Hinweis, dass im Zeitraum von drei Jahren bis zur ersten Rezertifizierung insgesamt 12 Ärzte aus den Bereichen Notaufnahme, Herzkatheterlabor und Intensivstation des zertifizierten CAC an einem ALS-Provider-Kurs des European Resuscitation Council (ERC) bzw. ACLS-Kurs der American Heart Association (AHA) teilgenommen haben müssen und dass im Rahmen der Rezertifizierung über die erfolgreiche Teilnahme ein Nachweis erbracht werden muss. Bei der Pflege gilt die gleiche Mindestanzahl an absolvierten Kursen, hier ist allerdings ein zertifizierter ILS-Provider-Kurs des ERC (alternativ ACLS-Kurs der AHA) ausreichend.

Dies dient dem mittelfristigen Ziel, mindestens einen Mitarbeiter mit Zertifikat im Cardiac Arrest Receiving Team (CART) 24/7 vorweisen zu können.

Für das Erstaudit ist somit kein Nachweis über die Kurse erforderlich. Wünschenswert ist dennoch, dass möglichst viele Mitarbeiter zertifizier- 
Abb. $1 \triangle$ Logo Cardiac Arrest Center

te Kurse (ausschließlich ALS, ILS oder alternativ ACLS) absolviert haben.

10. Verbindliches Ausbildungskonzept Zusätzlich zu den zu erbringenden Nachweisen über die Weiterbildungsbefugnisse der Mitglieder des Cardiac Arrest Zentrums und zu den ALS/ACLS- und ILS-Zertifikaten muss für jedes Cardiac Arrest Zentrum ein verbindliches Ausbildungskonzept für die Mitarbeiter erstellt und vorgelegt werden.

\section{Prozessqualität}

Zur Sicherstellung der gebotenen und adäquaten Prozessqualität sind detaillierte - und interdisziplinär und interprofessionell konsentierte - Standard Operating Procedures (SOP) für die folgenden Prozesse erforderlich:

1. SOP zur Übernahme von Notfallpatienten nach prähospitaler Reanimation [1]

2. SOP zur Schnittstellenkommunikation mit dem Rettungsdienst [1]

Die notwendige Kommunikation zwischen Rettungsdienst und Cardiac Arrest Zentrum beinhaltet die strukturierte und dokumentierte Notfallpatientenanmeldung, die Definition von Kommunikationswegen - ausdrücklich in beiden Richtungen - und die klare Festlegung von Verantwortlichkeiten. Ebenfalls hierunter abzubilden sind vorhandene oder zukünftige Komponenten telemedizinischer Technologien.

3. SOP zur Patientenübernahme durch ein Cardiac Arrest Receiving Team (CART). Hier soll insbesondere auch die Situation einer Übergabe bei noch laufender Reanimation Berücksichtigung finden [11].

4. SOP zur Notfalldiagnostik bei Notaufnahme nach Reanimation [1]

5. SOP zur Intensivtherapie einschließlich Temperaturmanagement [1]
6. SOP zu strukturiertem OutcomeAssessment/Therapieabbruch [1]

Dies beinhaltet strukturierte Regelungen zur unabhängigen Prognosebeurteilung durch qualifizierte Teams (fachneurologische Beurteilung), ggf. mit Einbindung eines lokalen Ethikkomitees.

7. SOP zum Angehörigengespräch [1]

8. SOP zu einer möglichen Organspende [1]

\section{Ergebnisqualität}

Der Nachweis einer systematischen und standardisierten Erfassung des Behandlungsverlaufs und des Outcomes bis zur Entlassung ([1]; überregionale Datenerfassung/überregionales Register, die/das grundsätzlich Benchmark erlaubt und auch die Möglichkeit zur Beteiligung an wissenschaftlichen Projekten bietet) ist für alle Patienten sicherzustellen. Datenschutzrichtlinien müssen beachtet werden.

Bei reanimierten STEMI-Patienten muss die Direktübergabe durch Notarzt/ Rettungsdienst im Katheterlabor überprüfbar dokumentiert werden [13].

Die Ergebnisse müssen beim Audit und Reaudit vorgestellt werden.

\section{Anwesende beim Audit}

Beim Audit vor Ort müssen folgende Personen anwesend sein:

- Der Leiter oder stellvertretende Leiter des CAC

- Leiter oder ein Stellvertreter der am CAC beteiligten Kliniken

- Ärztlicher Leiter Rettungsdienst oder Stellvertreter

\section{Rezertifizierung}

Aufgrund der zahlreichen Übergangslösungen soll das erste Reaudit nach 3 Jahren erfolgen.

Diese Regelungen sind mit einer Übergangsfrist von 4 Monaten ab 01.10.2021 gültig.
Korrespondenzadresse

Prof. Dr. med. K. H. Scholz

Medizinische Klinik I, Kardiologie u. Intensivmedizin, St. Bernward-Krankenhaus Hildesheim

Treibestraße 9, 31134 Hildesheim, Deutschland k.scholz@bernward-khs.de

\section{Einhaltung ethischer Richtlinien}

Interessenkonflikt. K.H. Scholz: Ehemals Vorstandsmitglied des Deutschen Rates für Wiederbelebung/ German Resuscitation Council (GRC); Initiator und Leiter des FITT-OHCA- und des FITT-STEMI-Studienprojekts. H.J. Busch: Mitglied des Exekutivkomitees des GRC und Co-Sprecher des Cardiac Arrest Zentrums Freiburg. N. Rott: Mitarbeiterin des GRC. B.W. Böttiger: Schatzmeister und Immediate Past Director Science and Research des European Resuscitation Council (ERC); Vorstandsvorsitzender des Deutschen Rates für Wiederbelebung/German Resuscitation Council (GRC); Mitglied im Präsidium der Deutschen Interdisziplinären Vereinigung für Intensiv- und Notfallmedizin (DIVI); Gründer der Deutschen Stiftung Wiederbelebung, Associated Editor des European Journal of Anaesthesiology (EJA); Mitherausgeber der Zeitschrift Resuscitation; Schriftleiter der Zeitschrift Notfall + Rettungsmedizin, Mitherausgeber der Zeitschrift Brazilian Journal of Anesthesiology. Für Vorträge hat er Honorare der folgenden Firmen erhalten: Forum für medizinische Fortbildung (FomF), Baxalta Deutschland GmbH, ZOLL Medical Deutschland $\mathrm{GmbH}, \mathrm{C}$. R. Bard GmbH, GS Elektromedizinische Geräte G. Stemple GmbH, Novartis Pharma GmbH, Philips GmbH Market DACH, Bioscience Valuation BSV $\mathrm{GmbH}$. N. Frey, M. Kelm und H. Thiele geben an, dass kein Interessenkonflikt besteht.

Für diesen Beitrag wurden von den Autoren keine Studien an Menschen oder Tieren durchgeführt. Für die aufgeführten Studien gelten die jeweils dort angegebenen ethischen Richtlinien.

\section{Literatur}

1. Scholz KH, Andresen D, Böttiger BW, Busch HJ, Fischer M, Frey N, Kelm M, Kill C, Schieffer B, Storm C, Thiele H, Gräsner JT (2017) Qualitätsindikatoren und strukturelle Voraussetzungen für CardiacArrest-Zentren-Deutscher Rat für Wiederbelebung/German Resuscitation Council (GRC). Notfal Rettungsmed. 2017;20:234-236

2. Scholz KH, Andresen D, Böttiger BW, Busch $\mathrm{HJ}$ Fischer M, Frey N, Kelm M, Kill C, Schieffer B, Storm C, Thiele H, Gräsner JT (2017) Qualitätsindikatoren und strukturelle Voraussetzungen für Cardiac-Arrest-Zentren-Deutscher Rat für Wiederbelebung/ German Resuscitation Council (GRC). Anaesthesist 2017;66:360-362

3. Scholz KH, Andresen D, Böttiger BW, Busch HJ, Fischer M, Frey N, Kelm M, Kill C, Schieffer B, Storm C, Thiele H, Gräsner JT (2017) Qualitätsindikatoren und strukturelle Voraussetzungen für Cardiac-Arrest-Zentren-Deutscher Rat für Wiederbelebung/ German Resuscitation Council (GRC). Med Klin Intensivmed Notfmed 2017;112:459-461

4. Scholz KH, Andresen D, Böttiger BW, Busch HJ, Fischer M, Frey N, Kelm M, Kill C, Schieffer B, Storm C, Thiele H, Gräsner JT (2017) Qualitätsindikatoren 
und strukturelle Voraussetzungen für CardiacArrest-Zentren-Deutscher Rat für Wiederbelebung/German Resuscitation Council (GRC). Der Kardiologe, 2017;11:205-208

5. Rott N, Scholz KH, Busch HJ, Frey N, Kelm M, Thiele H, Böttiger BW (2020) Cardiac Arrest Center Certification for out-of-hospital cardiac arrest patients successfully established in Germany. Resuscitation 156:1-3

6. Ibanez B, James S, Agewall S et al (2018) 2017 ESC Guidelines for the management of acute myocardial infarction in patients presenting with ST-segment elevation: The Task Force for the management of acute myocardial infarction in patients presenting with ST-segment elevation of the European Society of Cardiology (ESC). Eur Heart J39(2):119-177

7. Wijns W, Naber CK (2018) Reperfusion delay in patients with high-risk ST-segment elevation myocardial infarction: every minute counts, much more than suspected. Eur Heart J39:1075-1077

8. Monsieurs KG, Nolan JP, Bossaert LL et al (2015) European Resuscitation Council Guidelines for Resuscitation 2015: Section 1. Executive summary. Resuscitation 1:80

9. Sinning C, Ahrens I, Cariou A et al (2020) The cardiac arrest centre for the treatment of sudden cardiac arrest due to presumed cardiac cause-aims, function, and structure: position paper of the ACVC association of the ESC, EAPCI, EHRA, ERC, EUSEM and ESICM. Eur Heart J Acute Cardiovasc Care 9(S1):193-202

10. Semeraro F, Greif R, Böttiger BW et al (2021) European Resuscitation Council guidelines 2021: systems saving lives. Resuscitation 161:80-97

11. Busch HJ, Schmid B, Kron J, Fink K, Busche $C$, Danner T, Veits O, Gottlieb D, Benk C, Trummer G, Meyer-Först S, KoppS, Schwab W, Wengenmayer T, Biever P (2020) Freiburg Cardiac Arrest Receiving Team (CART): interdisciplinary solution for the acute management of non-traumatic out-ofhospital cardiac arrest. Med Klin Intensivmed Notfmed 115:292-299

12. Collet J-P, Thiele H, Barbato E et al (2021) 2020 ESC Guidelines for the management of acute coronary syndromes in patients presenting without persistent ST-segment elevation: The Task Force for the management of acute coronary syndromes in patients presenting without persistent STsegment elevation of the European Society of Cardiology (ESC). Eur Heart J 42:1289-1136

13. Scholz KH, Friede T, Meyer T, Jacobshagen $\mathrm{C}$, Lengenfelder B, Jung J, Fleischmann $C$, Moehlis $\mathrm{H}$, Olbrich HG, Ott R, Elsässer A, Schröder S, Thilo C, Raut W, Franke A, Maier LS, Maier SK (2020) Prognostic significance of emergency department bypass in stable and unstable patients with STsegmentelevation myocardialinfarction. EurHeart J Acute Cardiovasc Care 9(S1):34-44

\section{Quality indicators and structural requirements for Cardiac Arrest Centers-Update 2021. German Resuscitation Council (GRC)}

An update of the first description of quality indicators and structural requirements for Cardiac Arrest Centers from 2017 based on first experiences and certifications is presented. Criteria were adjusted, substantiated and in some parts redefined for feasibility in everyday clinical use.

\section{Keywords}

Postresuscitation care - Cardiac arrest center - Chain of survival - Out of hospital cardiac arrest . System saving lives 